\title{
Novel Low-Density Signature for Synchronous CDMA Systems Over AWGN Channel
}

\author{
Reza Hoshyar, Member, IEEE, Ferry P. Wathan, Student Member, IEEE, and Rahim Tafazolli
}

\begin{abstract}
Novel low-density signature (LDS) structure is proposed for transmission and detection of symbol-synchronous communication over memoryless Gaussian channel. Given $N$ as the processing gain, under this new arrangement, users' symbols are spread over $N$ chips but virtually only $d_{v}<N$ chips that contain nonzero-values. The spread symbol is then so uniquely interleaved as the sampled, at chip rate, received signal contains the contribution from only $d_{c}<K$ number of users, where $K$ denotes the total number of users in the system. Furthermore, a near-optimum chip-level iterative soft-in-soft-out (SISO) multiuser decoding (MUD), which is based on message passing algorithm (MPA) technique, is proposed to approximate optimum detection by efficiently exploiting the LDS structure. Given $\beta=K / N$ as the system loading, our simulation suggested that the proposed system alongside the proposed detection technique, in AWGN channel, can achieve an overall performance that is close to single-user performance, even when the system has $200 \%$ loading, i.e., when $\beta=2$. Its robustness against near-far effect and its performance behavior that is very similar to optimum detection are demonstrated in this paper. In addition, the complexity required for detection is now exponential to $d_{c}$ instead of $K$ as in conventional code division multiple access (CDMA) structure employing optimum multiuser detector.
\end{abstract}

Index Terms-Chip-level iterative SISO MUD, Gaussian channel, low-density signature, message passing algorithm, overloaded condition.

\section{INTRODUCTION}

$\mathbf{O}$ VER the last decades, code-division multiple access (CDMA) technique has been used by many commercial systems, IS-95 and UMTS to name a few. It formats several users to share a common communication channel simultaneously. Users' separation can be done by assigning codes, namely the spreading signatures, to each user uniquely. As consequences of this channel sharing, the interference: intersymbol interference (ISI) and/or multiple access interference (MAI), is inevitable in CDMA-based systems. Precisely, it is these interference that practically limits the performance of CDMA-based systems.

Manuscript received August 7, 2006; revised July 28, 2007. The associate editor coordinating the review of this manuscript and approving it for publication was Dr. Kostas Berberidis.

The authors are with the Mobile Communication Research Group, Centre for Communication Systems Research, University of Surrey, U.K. (e-mail: f.wathan@surrey.ac.uk).

Color versions of one or more of the figures in this paper are available online at http://ieeexplore.ieee.org.

Digital Object Identifier 10.1109/TSP.2007.909320
As interference-limited systems, logically, their performance should improve if the interference could be mitigated. Significant efforts have been made to address different aspects that might reduce the amount of interference and it include spreading signatures optimization, spreading strategy, intensive multiuser detection techniques, and the employment of forward error correction (FEC) coding scheme in the form of turbo multiuser decoding (MUD). However, since this paper will deal with uncoded systems, the FEC techniques will not be discussed further.

In communication of symbol-synchronous, uncoded CDMAbased systems over memoryless Gaussian channel, where this paper concentrates on, the optimal signatures are the orthogonal sets. Currently there are many orthogonalizing algorithms available, for example Householder and Gram-Schmidt algorithms [1], that can be used to construct orthogonal sets. However, it is impossible to obtain orthogonal sets in the condition where the number of active users $K$ is bigger than processing gain $N$, namely the overloaded condition. For that condition and without the luxury of optimum MUD techniques at the receiver, it has been well-studied in the literatures that the optimum sequences for symbol-synchronous memoryless channel are the ones that are derived to meet the Welch-bound-equality (WBE), which minimizes the variance of the MAI. The equality comes from the fact that the sum of the squared cross-correlations among $K$ signatures is equal to Welch's lower bound devised in [2]. The real-valued WBE-based techniques have been proposed in [3], [4], while the complex valued ones can be found in [5] and [6]. Another techniques to handle overloading (or oversaturated) condition are the family of hierarchy of orthogonal subsets (HoS) have been proposed in [7]-[9].

Another interesting attempts to optimize the signature sets is made by utilizing a feedback to jointly optimize the signature and the receiver as proposed in [10]-[12]. Furthermore, Users' grouping strategy has also caught our attention as proposed in [13] and its references therein.

Without channel information at the transmitter, the interference can, thence, be mitigated by using sophisticated multiuser detection techniques at the receiver. The optimum multiuser detector [14] yields the best performance in terms of the achievable probability of error yet its computational complexity that is exponential to $K$ prohibit its practical implementation. Later, numerous suboptimum low-complexity detectors have been proposed and the tradeoff between performance and complexity have been studied extensively. Some references and tutorial about multiuser detection can be found in [15]-[17]. In the class of linear detector, decorrelator [18], [19] and MMSE detector [20], [21] provide good performance with much lower 
complexity compared to their optimum counterparts. However, they fail to perform at satisfactory level under an overloaded condition as the desired (as well as the interferers') signal subspaces become rank-deficient. Furthermore, group multiuser detection strategy have been studied in [22]. Group multiuser detection can be implemented based on both decorrelator [22] and MMSE detector [23] and can be used to handle overloaded condition up to some extent. A generalized and improvised form of group MUD for overloaded condition have been evaluated in [24], where a decision-feedback detector proposed in [25] and [26] is also considered for users grouping strategy.

As for class of nonlinear detector, e.g., multistage detection [27], successive interference cancellation (SIC) [28] and parallel interference cancelation (PIC) [29], while they offer a very simple detection process yet the performance is highly dependent on the first estimate being fed to the IC detector. More recently, iterative multiuser detection have also been recommended by using probabilistic data association (PDA) [30] and have been shown to achieve close to optimum detector. Yet again, its performance degrades rapidly in overloaded condition.

Recently, another investigation have been conducted and the author has concluded that to achieve the theoretical capacity limit, the whole channel bandwidth should be devoted for coding. Taking that into account, interleave-division multiple access (IDMA) [31] have been proposed, whereas in contrast with CDMA, IDMA distinguishes its users by assigning a unique interleaver for each user and, thus, removing the need of spreading process. The reason behind this is that the spreading only adds redundancy to distinguish user without giving any coding gain. By employing a simple convolutional code as FEC and with turbo-style processing at the receiver, it results in a very good system performance that achieve near capacity limit. Then, the performance will be too dependent on the FEC codes being employed.

From the above discussions, it is the fact that in CDMA systems the interference is sometimes unavoidable at the receiver due to nonorthogonality of the signatures and/or the effect of the channel. The question is that, if we are to allow some interference to exist then what is the best possible to manage it such that it can play into our advantage.

In conventional CDMA structure, when transmitting over memoryless symbol-synchronous channel, every sample (at chip-rate, then it will be named chip for later discussion) of the received signal will carry the contribution from all users in the system. In other words, each user sees the contribution from all other users at every received chip. If the cross-correlation matrix of the signatures follows some format, optimum MUDs can be deployed with lower complexity, e.g., tree detector [7], however, its complexity is still much higher than that of linear-detectors. Recall the result from [7], [31] and the resultant signature matrices derived from WBE-bound [5], we reckon that it is not very necessary to set nonzero-values to all of the signature chips. Our investigation suggests that if an intelligent arrangement can be made to change the system's interference pattern, a performance improvement can be achieved.

Furthermore, inspired by our investigation above and the recent success of the low-density parity check (LDPC) codes, devised in [32], we propose a novel low-density signature
(LDS) structure that follows the parity-check matrix in LDPC codes. Notice that, unlike [10] that projects a signature into lower-rank subspaces and may, depending on the optimization, switch off some of the chips, the LDS structure intentionally switches a large fraction of the spreading chips off. Moreover, by switching-off those chips, we can facilitate an affordable close to optimum MUD that is based on message-passing algorithm (MPA). Therefore, to complement the proposed LDS structure, it is necessary to submit a new iterative chip-level MPA-based detection to approximate the optimum maximum $a$ posteriori (MAP) detector. While the proposed iterative detection technique has similar features as the sum-product algorithm explained in [33], the application of this kind of iterative processing in uncoded systems is novel (for coded CDMA using conventional structure, the similar iterative frame-by-frame processing to exploit the FEC-powered turbo-MUD property can be found in [34]) and to best of our knowledge has never been considered.

Our simulations showed that the proposed LDS structure complemented with the proposed iterative detector can achieve a robust near single-user performance with the loading of up to $200 \%$ with an affordable computational complexity compared to conventional structure employing optimum multiuser detection.

This paper is organized as follows. Section II presents a canonical synchronous CDMA system model. Then, a novel LDS structure will be explained in more detail in III, where the design criterions and an example will be presented. The novel chip-level iterative receiver is discussed in Section IV. Later, the simulation results are presented and analyzed in Section V. It includes several scenarios, as well as the performance against near-far effect problem. Furthermore, the convergence rate of the iterative receiver is given. Finally, we conclude this paper in Section VI.

Throughout this paper, we use the following notations. A vector and a matrix will be represented by lower-caps and capital, respectively, both in bold case. All vector is defined as a column vector. Constants and variables are denoted in capital and lower-caps, correspondingly. Superscripts $T$ and $H$ represent transpose and Hermitian version of the vector and/or matrix, respectively. The symbol $\sim$ and $\propto$ constitute "is distributed according to" and "is proportional to," respectively.

\section{SynChronous CDMA System Model}

Consider a symbol-synchronous uncoded CDMA system with $K$ users and processing gain of $N$. Let $x_{k}$ and $\mathbb{X}_{k}$ be, respectively, the transmitted symbol with period of $T_{s}$ and the constellation alphabet, from which $x_{k}$ will take its value, for user $k$. Thus, the modulated symbol $x_{k}$ is formed by mapping a sequence of independent information bits $\mathbf{b}_{k} \in \mathbb{F}_{2}^{m_{k}}$ to $\mathbb{X}_{k}$ and can be represented by the function $\phi_{k}: \mathbb{F}_{2}^{m_{k}} \rightarrow \mathbb{X}_{k}$, where $m_{k}$ is the number of information bits per symbol. Given $a \in \mathbb{X}_{k}$, $\mathbb{X}_{k}$ is then normalized to have a unit average energy such that

$$
\frac{1}{\left|\mathbb{X}_{k}\right|} \sum_{a \in \mathbb{X}_{k}}|a|^{2}=1
$$




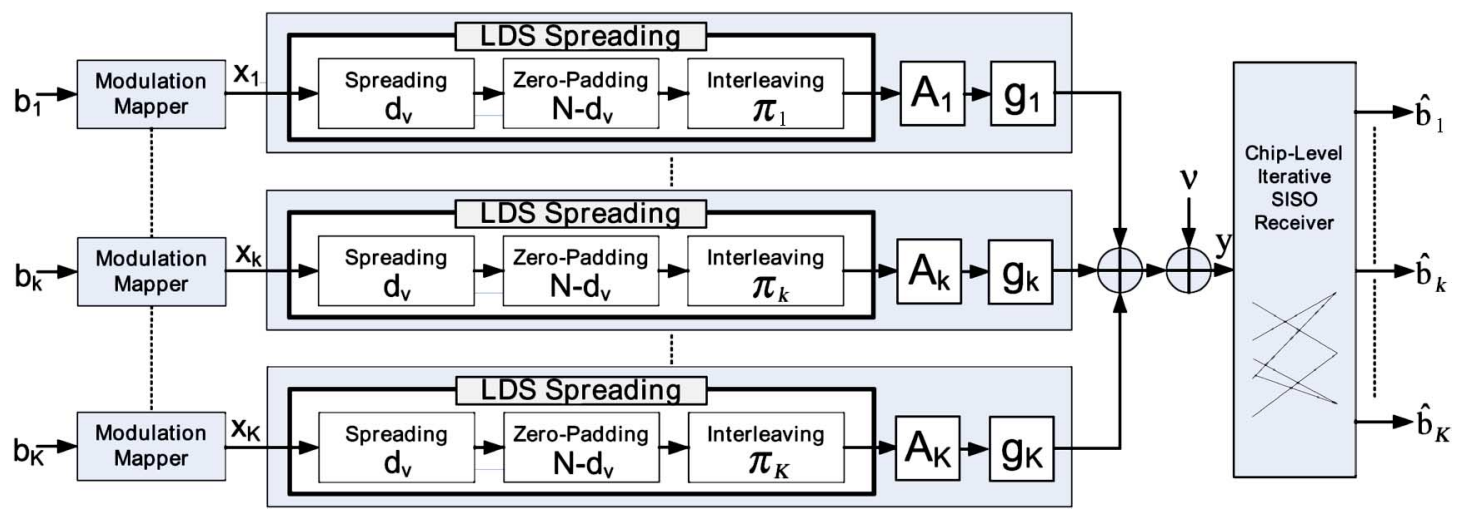

Fig. 1. LDS CDMA system model.

where $\left|\mathbb{X}_{k}\right|=2^{m_{k}}$ denotes the cardinality of $\mathbb{X}_{k}$. Without loss of generality and for notation brevity, all users are assumed to take their symbol from the same constellation alphabet, i.e., $\mathbb{X}=$ $\mathbb{X}_{k}, \forall k=1, \ldots, K$.

For an uplink transmission over memoryless Gaussian channel, where this paper concentrates on, the signal is sufficiently detected on symbol-by-symbol basis. Let $\mathbf{y}=\left[y_{0}, \ldots, y_{N-1}\right]^{T}, \mathbf{h}_{k}=\left[h_{0, k}, \ldots, h_{N-1, k}\right]^{T}$ and $\mathbf{v}=\left[v_{0}, \ldots, v_{N-1}\right]^{T}$ be the received, effective signature for user $k$ and the noise vector of size $N$, respectively, which are formed by stacking $N$ successive chips together. Assuming that all users are perfectly aligned in time, the received signal, which is the superimposed version of the transmitted signals from all users, can be written in discrete-form as

$$
\begin{aligned}
\mathbf{y} & =\sum_{\substack{k=1\\
}}^{K} \mathbf{h}_{k} x_{k}+\mathbf{v} \\
& =\mathbf{H x}+\mathbf{v}
\end{aligned}
$$

where $\mathbf{H}=\left[\mathbf{h}_{1} \ldots \mathbf{h}_{K}\right], \mathbf{x}=\left[x_{1}, \ldots, x_{K}\right]^{T}$ and $\mathbf{v} \sim$ $\mathcal{C N}\left[0, \sigma^{2} \mathbf{I}\right]$ denote effective received signature matrix, transmitted symbols and noise vectors, respectively. The effective receive signature $\mathbf{H}$ can also be decomposed as

$$
\mathbf{H}=\mathbf{A G S}
$$

where $\mathbf{A}=\operatorname{diag}\left(A_{1}, \ldots, A_{K}\right), \mathbf{G}=\operatorname{diag}\left(g_{1}, \ldots, g_{K}\right)$, and $\mathbf{S}=\left(\mathbf{s}_{1}, \ldots, \mathbf{s}_{K}\right)$ represent the users' transmit gain, the corresponding channel gain and the spreading signature matrices for each user, respectively. From (1) and (3), the receive spreading signature for user $k$ is denoted as $\mathbf{h}_{k}=A_{k} * g_{k}\left[s_{1, k}, s_{2, k}, \ldots, s_{N, k}\right]$, where $s_{n, k} \in \mathbb{C}$. In particular, the received signature at chip $n$ of user $k$ is $h_{n, k}=A_{k} \cdot g_{k} \cdot s_{n, k}$.

\section{NOVEL LDS STRUCTURE}

The spreading sequences are assigned uniquely to each user and are fully transparent to receiver. In conventional structure, each element of the spreading sequences takes, usually, nonzero values, which is optimized to certain constraints, e.g., good auto- and/or cross-correlation. Then, from (1) the received signal at chip $n$ with conventional structure can be denoted as

$$
y_{n}=\sum_{k=1}^{K} h_{n, k} x_{k}+\nu_{n}, \quad n=0, \ldots, N-1
$$

From (4), it is easy to see in conventional structure that, at received chip $y_{n}$, each user $k$ will see the interference coming from other $K-1$ users.

Instead of optimizing the $N$-chips signatures, we propose a novel LDS structure that intentionally arranges each user to spread its data over a small number of chips and then zeropadding is done such that the processing gain is still $N$. The spreading sequences are then interleaved uniquely for each user such that the resultant signatures matrix become very sparse. This proposed LDS system model is depicted in Fig. 1.

The interleaving process is so designed that at each received chip there exists a contribution of, instead of all users, only a small number of users. Consequently, the interference pattern being seen by each user is different. Let $r_{n, k}=h_{n, k} x_{k}$ be the received signal at the $n$th chip due to user $k$. It can be seen that, for the LDS structure, at the chip-level, user 1 sees an interference coming from different users at different chips. Furthermore, the interference from several chips, over which user 1 does not send its information, can be simply neglected in the detection process. The LDS philosophy is that if a fraction of signal of some user is superimposed by a fraction of signals coming from a relatively small number of interferers, then the search-space should be smaller and, hence, more affordable detection technique can be used to recover the corrupted fraction of signal. Moreover, apart from more convenient implementation of the detector, LDS structure also is benefited from having an intrinsic interference diversity by avoiding strong interferers to corrupt all chips of a user.

Let $d_{v}$ and $d_{c}$ be the maximum number of chips over which a single user is allowed to spread its data and the maximum number of users that is allowed to interfere within a single chip, respectively. The new spreading sequences for each user will, then, have a maximum of $d_{v}$ nonzero values and $N-d_{v}$ zerovalues. In order to make this new structure clearer, we introduce 
an indicator vector $\mathbf{f}_{k} \in \mathbb{F}_{2}^{N}$, where its $n$th element is denoted by $f_{n, k}$ and is given by

$$
f_{n, k}=\left\{\begin{array}{lll}
0, & \text { then } & s_{n, k}=0 \\
1, & \text { then } \quad s_{n, k} \neq 0
\end{array} ; \quad k=1, \ldots, K .\right.
$$

The position of 1's in the indicator vector $\mathbf{f}_{k}$ denotes the position, after interleaving process, over which user $k$ may spread its data. These arrangements can be also seen as chip-hopping structure where the position of 1's in $\mathbf{f}$ represents the time (relative to the first chip) when user $k$ is allowed to transmit a copy version of its data.

Indicator matrix is given by $\mathbf{F}=\left[\mathbf{f}_{1}, \ldots, \mathbf{f}_{K}\right]$. The set of position of 1's in the $n$th row of $\mathbf{F}$ denotes the set of users that contribute their data at the $n$th chip, while its $k$ th column represents the set of chips over which user $k$ spreads its data. Note that the 1's components of the indicator matrix $\mathbf{F}$ represents the nonzero components of matrix $\mathbf{H}$ and the nonzero components of matrix $\mathbf{H}$ can take any complex-valued.

Let $\xi_{n}$ and $\zeta_{k}$ be set of 1's position in the $n$th row and in the $k$ th column, respectively, then we can modify (4) as

$$
\begin{aligned}
y_{n} & =\sum_{k \in \xi_{n}} h_{n, k} x_{k}+\nu_{n} \\
& =\mathbf{h}^{[n] T} \mathbf{x}^{[n]}+\nu_{n}
\end{aligned}
$$

where $\mathbf{X}^{[n]}$ and $\mathbf{h}^{[n]}$ denote the vector stacking the symbols transmitted by every user participating in chip index $n$ and their corresponding fraction of effective receive signature values, respectively. Note that, in LDS structure, we still assume the signature waveforms for all users are supported within one symbol interval and are normalized that its power spectral density is one, i.e., $\left\|\mathbf{s}_{k}\right\|=1, \forall k=1, \ldots, K$.

Rule 1 (Requirements for LDS Indicator Matrix): LDS indicator matrix is designed with the following requirements.

1) The maximum number of 1 's in each column $d_{v}$ must be sufficiently smaller than $N$.

2) The maximum number of 1's in each row $d_{c}$ must be sufficiently smaller than $K$.

3) Full connectivity on the associated Bipartite graph of the indicator matrix.
From the aforementioned requirements, we can easily see that it is very similar to the requirements of LDPC matrix. Unlike LDPC matrix, the proposed LDS structure do not strictly prevent the associated Bipartite graph of the indicator matrix from having short cycles, therefore, any LDPC matrix can be used as our LDS matrix. The LDS matrices being used in this paper are either constructed by manually or from the LDPC matrices [35]. However, another constraint is imposed as stated in the third requirement, i.e., full-connectivity. It means that each and every node in the associated graph should be able to reach other nodes. This constraint will pave way to full-cooperative processing to be carried out iteratively.

The LDS indicator matrix $\mathbf{F}$ is created to meet with the required $N, K$, and $d_{v}$. As it is difficult to find the relationship between all that three variables mentioned earlier for irregular structure, we can find their relationship for (quasi)-regular ${ }^{1}$ structure, i.e., $\min d_{c}=\lfloor K / N\rfloor d_{v}$.

Furthermore, if the LDS indicator matrix has the same number of 1's in each column, i.e., $d_{v}$ and also the same number of 1 's in each row, i.e., $d_{c}$, but $d_{c}$ is not necessarily equal to $d_{v}$, then the structure is called regular LDS, otherwise irregular LDS. To explain the LDS structure in more detail, in the following, we present an example of LDS structure and some parameters we have introduced in this paper.

Example 1: Regular LDS structure is designed to serve up to 16 users with processing gain of 12 chips. The corresponding indicator vector is given by $\mathbf{F}_{12 \times 16}$ with $d_{v}=3$ and $d_{c}=4$. (See the matrix at the bottom of the page.)

From $\mathbf{F}_{12 \times 16}$, we create a set of users that are interfering to each other in third chip, i.e., $\xi_{3}=\{2,5,7,10\}$ and a set of position over which user 11 will spread its data, i.e., $\zeta_{11}=$ $\{4,11,12\}$. Furthermore, while observing ninth chip, the corresponding effective received signature vector is given by $\mathbf{h}^{[9]}=$ $\left[h_{9,1}, h_{9,7}, h_{9,14}, h_{9,16}\right]^{T}$.

\section{Chip-Level Iterative Soft-IN-Soft-Out (SISO) MulTiUSER DECODING}

The optimum multiuser detection problem can be solved by finding the maximum joint $a$ posteriori probability mass function (pmf) of all users' transmitted symbols, i.e., by using MAP

${ }^{1}$ when $d_{v}$ are the same for all users, $d_{c}$ may vary in the minority of the chips

$$
\mathbf{F}_{12 \times 16}=\left[\begin{array}{cccccccccccccccc}
0 & 0 & 1 & 0 & 0 & 0 & 0 & 1 & 0 & 1 & 0 & 0 & 1 & 0 & 0 & 0 \\
0 & 0 & 0 & 1 & 0 & 0 & 1 & 0 & 0 & 0 & 0 & 0 & 1 & 0 & 1 & 0 \\
0 & 1 & 0 & 0 & 1 & 0 & 1 & 0 & 0 & 1 & 0 & 0 & 0 & 0 & 0 & 0 \\
0 & 0 & 0 & 1 & 0 & 1 & 0 & 0 & 0 & 0 & 1 & 0 & 0 & 1 & 0 & 0 \\
0 & 0 & 1 & 0 & 0 & 0 & 0 & 0 & 1 & 0 & 0 & 0 & 0 & 0 & 1 & 1 \\
1 & 0 & 0 & 0 & 0 & 1 & 0 & 0 & 0 & 1 & 0 & 0 & 0 & 0 & 1 & 0 \\
0 & 0 & 0 & 1 & 0 & 0 & 0 & 1 & 1 & 0 & 0 & 1 & 0 & 0 & 0 & 0 \\
0 & 1 & 0 & 0 & 0 & 1 & 0 & 0 & 0 & 0 & 0 & 1 & 0 & 0 & 0 & 1 \\
1 & 0 & 0 & 0 & 0 & 0 & 1 & 0 & 0 & 0 & 0 & 0 & 0 & 1 & 0 & 1 \\
0 & 0 & 1 & 0 & 1 & 0 & 0 & 0 & 0 & 0 & 0 & 1 & 0 & 1 & 0 & 0 \\
0 & 1 & 0 & 0 & 0 & 0 & 0 & 0 & 1 & 0 & 1 & 0 & 1 & 0 & 0 & 0 \\
1 & 0 & 0 & 0 & 1 & 0 & 0 & 1 & 0 & 0 & 1 & 0 & 0 & 0 & 0 & 0
\end{array}\right] .
$$


detection with excessive search. It will minimize the probability that not all users are detected correctly or, in other words, it will minimize the average BER for all users. However, employing the optimum MAP to the proposed LDS CDMA systems gives no complexity reduction over the conventional CDMA systems, which is one of the intentions of the proposed LDS structure.

Therefore, in this section we propose and derive a novel suboptimum iterative chip-level SISO multiuser detection, which is based on MPA principle, to exploit the LDS structure efficiently. This technique will iteratively approximate the global optimum MAP detection by factorizing it into a product of simpler local observations and, then, combining it at the later stage. The factor-graph representation of the factorized optimum MAP detection will be constructed to give more insight detail into the proposed iterative multiuser detection technique.

\section{A. Optimum MAP Detection on LDS Structure}

Given $\mathbf{y}$ as the observation and the assumption that $\mathbf{H}$ is available at the receiver, the joint optimum MAP detection will estimate $\hat{\mathbf{X}}$ that maximizes the joint a posteriori pmf of the transmitted symbols $\mathbf{x}$, i.e., $p(\mathbf{x} \mid \mathbf{y})$, and can be written as

$$
\hat{\mathbf{x}}=\arg \max _{\mathbf{x} \in \mathbb{X}^{K}} p(\mathbf{x} \mid \mathbf{y})
$$

The transmitted symbol $x_{k}$ can also be estimated by maximizing its a posteriori pmf, which can be found by calculating the marginal of the joint a posteriori pmf function defined in (7) with respect to $x_{k}$ and can be written as

$$
\hat{x}_{k}=\arg \max _{a \in \mathbb{X}} \sum_{\substack{\mathbf{x} \in \mathbb{X}^{K} \\ x_{k}=a}} p(\mathbf{x} \mid \mathbf{y}), \quad \forall k
$$

and by using Bayes' rule, we have the following:

$$
\begin{aligned}
p(\mathbf{x} \mid \mathbf{y}) & =\frac{p(\mathbf{y} \mid \mathbf{x}) P(\mathbf{x})}{P(\mathbf{y})} \\
& \propto p(\mathbf{y} \mid \mathbf{x}) P(\mathbf{x})
\end{aligned}
$$

where $P(\mathbf{x})=\prod_{k=1}^{K} P\left(x_{k}\right)$ and $P(\mathbf{y})=\sum_{x \in \mathbb{X}^{k}} p(\mathbf{y} \mid \mathbf{x}) P(\mathbf{x})$ are the joint a priori pmf of all users' symbols assuming that they are independent to each other and the pdf of the received signal, respectively.

Furthermore, with the assumption that the noise vector is identically independently distributed (i.i.d.) and is uncorrelated with the transmitted symbols, we are allowed to factorize

$$
p(\mathbf{y} \mid \mathbf{x})=\prod_{n=1}^{N} p\left(y_{n} \mid \mathbf{x}\right)
$$

where $p\left(y_{n} \mid \mathbf{x}\right)$ is the channel observation function at chip $y_{n}$. Interestingly, since, with LDS structure, only maximum of $d_{c}$ users are allowed to interfere to each other, therefore, the calculation of this function can be simplified and is given by

$$
p\left(y_{n} \mid \mathbf{x}\right)=p\left(y_{n} \mid \mathbf{x}^{[n]}\right) .
$$

Furthermore, by combining (9), (10), and (11) into (8) and removing all the unnecessary constant scalars, we can write

$$
\hat{x}_{k}=\arg \max _{a \in \mathbb{X}} \sum_{\substack{\mathbf{x} \in \mathbb{X}^{K} \\ x_{k}=a}} P(\mathbf{x}) \prod_{n \in \zeta_{k}} p\left(y_{n} \mid \mathbf{x}^{[n]}\right)
$$

From (12), we can see that the proposed LDS structure allows us to translate the MAP detection problem into the marginalize product of functions (MPF) [33] problem much more efficiently. While the same translation can be done for conventional receiver, there is no obvious advantage of doing that in order to make the detection less-complex as each chips from a user sees the interference from other users.

\section{B. Factor Graph Representation}

A class of optimum multiuser detection described previously for the proposed LDS structure can be represented by its factor graph $\mathcal{G}(\mathcal{U}, \mathcal{C})$ [33], which is the bipartite graph representation of its factorization defined in (12). In this factor graph, the transmitted symbols $x_{k}, k=1, \ldots, K$ and the chip observation $y_{n}$, $n=1, \ldots, N$ are denoted as variable nodes $u \in \mathcal{U}$ and function nodes $c \in \mathcal{C}$, respectively.

Let edge $e_{k, n}$ be the edge that connects a function node $c_{n}$ to a variable node $u_{k}$. At the function node $c_{n}$, the local channel observation at chip $y_{n}$ is made and is given by

$$
p\left(y_{n} \mid \mathbf{x}^{[n]}\right)=\frac{1}{\sqrt{2 \pi} \sigma} \exp \left(-\frac{1}{2 \sigma^{2}}\left\|y_{n}-\mathbf{h}^{[n] T} \mathbf{x}^{[n]}\right\|^{2}\right) \text {. }
$$

With the LDS structure, it is easy to see in $\mathcal{G}(\mathcal{U}, \mathcal{C})$ that each variable node $u_{k}$ is connected only to $d_{v}$ function nodes $c_{n}$, $\forall n \in \zeta_{k}$ and that each function node $c_{n}$ is connected only to $d_{c}$ variable nodes $u_{k}, \forall k \in \xi_{n}$. Fig. 2 depicts an example of factorgraph representation of the LDS structure used in Example 1

\section{C. $M P A$}

As shown earlier, by employing the proposed LDS structure, we can translate the joint MAP detection problem into an MPF problem as shown in (12), where each function is much simpler to resolve. However, solving this MPF problem with brute-force still necessities exponential complexity.

Fortunately, there exists a general algorithm to iteratively approximate the solution of this MPF problem that is by applying an MPA onto the underlying factor-graph. This fact has motivated us to propose a novel suboptimum iterative chip-level MUD that is based on the generic MPA, i.e., sum-product algorithm(SPA) that is used for the decoding of LDPC. While the proposed iterative detection technique has similar feature as 


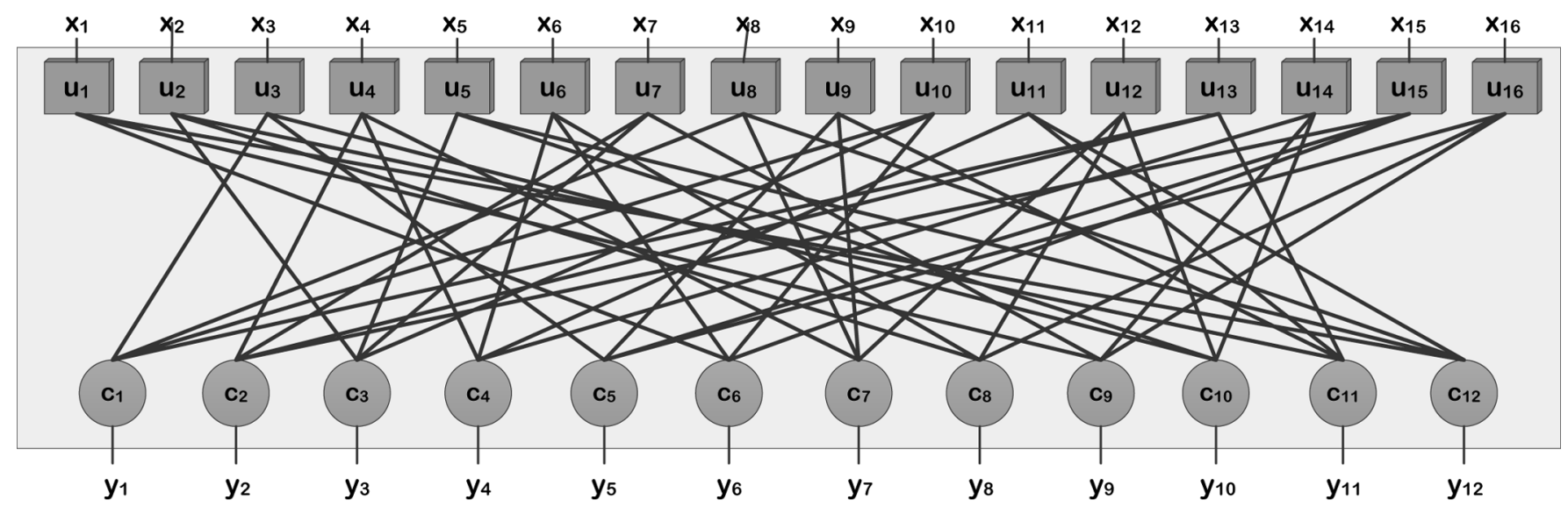

Fig. 2. Factor graph representation of the joint MAP detection for LDS scheme.

the one explained in [33], the application of this kind of iterative processing in uncoded systems is nove ${ }^{2}$ and to best of our knowledge, MPA algorithm for symbol-by-symbol processing has never been considered. Furthermore, our proposed iterative detector work on symbol-level instead of bit-level as shown in [33].

Operating on the factor-graph $\mathcal{G}(\mathcal{C}, \mathcal{U})$, the proposed iterative MUD works as follow. The messages are updated by iteratively exchanging them between function and variable nodes along the respective edges. Those messages are the soft-values that represent the inference or the reliability of the symbol associated to each edge. When the factor-graph has no cycles, after some iterations, the messages can be used to do exact inferences to each symbol $x_{k}$ [33]. However, when it contains cycles, the local computations, hence, become an iterative algorithm, which hopefully converges to a good approximation of $x_{k}$.

Remark 1 (Representation of a Message): For antipodal modulation, e.g., BPSK, log-likelihood ratio (LLR) value of the symbol can be used to represent the message. However, for higher order modulation, the message being exchanged must be in the form of a vector of size $|\mathbb{X}|$ comprising the reliability values for each of the possible values taken from the symbol constellation alphabet $\mathbb{X} .^{3}$ Note that, the message must be normalized such that the sum of all probability values for all alphabet symbols is one.

It is important to note that in the proposed detection technique, the messages that are exchanged along the edges during iteration contain only the extrinsic information as suggested for SPA in [36]. The message received from one edge cannot be used to update the message to be transmitted onto that edge.

Rule 2 (Updating a Message): Let edge $e_{n, k}$ the edge of observation. The message being sent from function node $c_{n}$ onto edge $e_{k, n}$ is the product ${ }^{4}$ of the messages received from edges

\footnotetext{
${ }^{2}$ the similar iterative frame-by-frame processing to exploit the FEC-powered turbo-MUD property has been used for coded-CDMA systems. Interested reader is referred to [34]

${ }^{3}$ The message can even be represented to vector of size $|\mathbb{X}|-1$ by taking one value as reference to others.

${ }^{4}$ If the message is represented by its logarithmic values of its probability values, then the summation operation shall be used instead of product operation
}

$e_{n, l}, \forall l \in \zeta_{n} \backslash k$ with the local function at $c_{n}$ and being summarized for the variable associated with the edge, i.e., $x_{k}$. Similarly, the variable node $u_{k}$ will send a message, which is the product of the messages received from edges $e_{m, k}, \forall m \in \xi_{k} \backslash n$, onto edge $e_{n, k}$.

For notation brevity and simplicity, throughout this paper, BPSK will be used as the modulation scheme and, therefore, $x_{k} \in\{+1,-1\}$ and the LLR values are sufficiently used to constitute the messages. The extension for higher order modulation is, however, straightforward and, therefore, it will not be presented.

Let $\ell_{c_{n} \leftarrow u_{k}}$ and $\ell_{c_{n} \rightarrow u_{k}}$ be the message sent along edge $e_{n, k}$ from variable node $u_{k}$ and function node $c_{n}$, respectively. The message $\ell_{c_{n} \leftarrow u_{k}}$ gives an updated inference of $x_{k}$ based on the observation taken at chips $y_{m}, \forall m \in \xi_{k} \backslash n$. To comply with Rule 2, we introduce a subscript "ext" to some pdfs to denote that only the extrinsic information should be used to calculate those particular pdfs. Therefore, we can write $\ell_{c_{n} \leftarrow u_{k}}$ as follows:

$$
\begin{aligned}
\ell_{c_{n} \leftarrow u_{k}} & =\log \frac{P_{\mathrm{ext}, n}\left(x_{k}=+1\right)}{P_{\mathrm{ext}, n}\left(x_{k}=-1\right)} \\
& =\sum_{m \in \xi_{k} \backslash n} \ell_{c_{m} \rightarrow u_{k}} .
\end{aligned}
$$

By using the message formulated in (14) and we can represent

$$
P_{\text {ext }, n}\left(x_{k}\right)=\lambda_{n, k} \exp \left(\frac{x_{k}}{2} \ell_{c_{n} \leftarrow u_{k}}\right)
$$

where $\lambda_{n, k}$ is chosen such that $P_{\mathrm{ext}, n}\left(x_{k}=+1\right)+P_{\mathrm{ext}, n}\left(x_{k}=\right.$ $-1)=1$.

At the function node $c_{n}$, the inference of $x_{k}$ is updated and is given by

$$
\begin{aligned}
\ell_{c_{n} \rightarrow u_{k}} & =\log \frac{p_{\text {ext }, n}\left(x_{k}=+1 \mid y_{n}, \mathbf{x}^{[n]} \backslash x_{k}\right)}{p_{\text {ext }, n}\left(x_{k}=-1 \mid y_{n}, \mathbf{x}^{[n]} \backslash x_{k}\right)} \\
& =\log \frac{p_{\text {ext }, n}\left(y_{n} \mid \mathbf{x}^{[n]}, x_{k}=+1\right) P\left(\mathbf{x}^{[n]} \backslash x_{k}\right)}{p_{\text {ext }, n}\left(y_{n} \mid \mathbf{x}^{[n]}, x_{k}=-1\right) P\left(\mathbf{x}^{[n]} \backslash x_{k}\right) .}
\end{aligned}
$$


By using Bayes' rule as in (9), the second equality is formed and by following rule 2 , then the a priori pmf of $x_{k}$ is not included in the computation of a posteriori pmf of $x_{k}$.

Combining (13), (14), and (15) into (16), we can write a complete message being sent from function node $c_{n}$ onto edge $e_{n, k}$ shown in (17) and (18) at the bottom of the page. After the message coming to variable nodes $u_{k}, k=1, \ldots, K$ have converged or the number of maximum iteration has been met, the variable nodes will use all message received from all connected edges to calculate the final estimated inference for symbols $x_{k}$, $k=1, \ldots, K$ and, akin to (15), is given by

$$
P\left(x_{k}= \pm 1\right)=\lambda_{k} \exp \left(\frac{x_{k}}{2} \sum_{n \in \zeta_{k}} \ell_{c_{n} \rightarrow u_{k}}\right), \quad k=1, \ldots, K
$$

where $\lambda_{k}$ is chosen so as $P\left(x_{k}=+1\right)+P\left(x_{k}=-1\right)=1$.

The max $^{\star}$ operation, as suggested in [37], used in (18), is a numerically stable operation and is defined as follows:

$$
\begin{aligned}
\max ^{\star}(a, b) & =\log (\exp (a)+\exp (b)) \\
& =\max (a, b)+\log (1+\exp (-|a-b|)) .
\end{aligned}
$$

The second term of (20) expresses that the $\max ^{\star}$ operation can be represented as the max operation plus a correction term. In the high SNR region, that correction term becomes very small, therefore max operation is enough to represent (20).

\section{Simulation Results}

In this section, we present the simulation performance of LDS-CDMA system with different LDS schemes.

1) Overloaded with $133 \%$ load: Regular LDS $12 \times 16$ with $d_{v}=3$

2) Overloaded with $133 \%$ load: Regular LDS $60 \times 80$ with $d_{v}=3$

3) Overloaded with $133 \%$ load: Irregular LDS $100 \times 133$ with $d_{v}=3$

4) Overloaded with $150 \%$ load: Irregular LDS $48 \times 72$ with $d_{v}=3$

5) Overloaded with $200 \%$ load: Regular LDS $12 \times 24$ with $d_{v}=3$.
Note that all simulations for LDS structure have been done by using the same spreading signatures associated for each of the aforementioned regular LDS schemes. Otherwise stated, novel near-optimum chip-level iterative SISO MAP-based algorithm, with maximum of 15 iterations, ${ }^{5}$ is deployed at the receiver. BPSK modulation is used throughout the simulation and users are transmitting their data with, otherwise stated, equal average received SNR. LDS $N \times K$ denotes an LDS structure that serves up to $K$ users by using processing gain of $N$ chips. Each user's data will be phase-rotated randomly uniquely each time it is transmitted. By doing so, we also check the system robustness against phase rotation effect in case of practical memoryless Gaussian channel.

\section{A. Small-System and Large-System Loading}

Here, we compare the spreading signatures for conventional structure derived from several signature optimization techniques for overloading condition and the proposed LDS structure, in terms of bit error rate (BER) for the system with $N=12$ and $K=16$. The detection being used are the MMSE-based PPIC and the proposed iterative chip-level detector with maximum iteration (or stages) of 15. The MMSE detector will give an unconstrained MMSE estimate of the transmitted symbols and PPIC will smooth it further with the smoothing-coefficient varies in ascending-order from 0.6 to $0.8^{6}$ with the same stepsize.

Fig. 3(a) shows that the random signature with LDS structure performed better than the one with conventional structure. In addition, by using the optimized signatures, the conventional structure performed better than the LDS structure using random signatures. Furthermore, if the LDS structure is employed using the best-found ${ }^{7}$ signatures, interestingly, it outperformed the ones with optimization. Yet the performance of the LDS structure deploying best-found signatures with MMSE-PPIC detector became saturated after $E_{b} / N 0=14 \mathrm{~dB}$. Furthermore, when the proposed structure is complemented with the proposed chip-level detector, i.e., Chip-level(CL)-MAP (with the

${ }^{5}$ No stopping criterion is implemented, therefore the system stops only after reaching the maximum iterations

${ }^{6}$ It is assumed that as the iteration goes further, the estimated value become more reliable.

${ }^{7}$ The best-found signatures for each of the LDS schemes were found by trialand-error.

$$
\begin{aligned}
& \ell_{c_{n} \rightarrow u_{k}}=\log \frac{\sum_{\substack{\mathbf{x}^{[n]} \in \mathbf{X}_{c} \\
x_{k}=+1}} \exp \left(\sum_{l \in \zeta_{n} \backslash k} \frac{x_{l}}{2} \ell_{c_{n} \leftarrow u_{l}}-\frac{1}{2 \sigma^{2}}\left\|y_{n}-\mathbf{h}^{[n] T} \mathbf{X}^{[n]}\right\|^{2}\right)}{\sum_{\substack{\mathbf{x}^{[n]} \in \mathbf{X}_{c} \\
x_{k}=-1}} \exp \left(\sum_{l \in \zeta_{n} \backslash k} \frac{x_{l}}{2} \ell_{c_{n} \leftarrow u_{l}}-\frac{1}{2 \sigma^{2}}\left\|y_{n}-\mathbf{h}^{[n] T} \mathbf{X}^{[n]}\right\|^{2}\right)} \\
& =\max _{\substack{\mathbf{x}[n] \in \mathbf{x}^{d_{c}} \\
x_{k}=+1}} \star\left(\sum_{\substack{l \in \zeta_{n} \backslash k\\
}} \frac{x_{l}}{2} \ell_{c_{n} \leftarrow u_{l}}-\frac{1}{2 \sigma^{2}}\left\|y_{n}-\mathbf{h}^{[n] T} \mathbf{x}^{[n]}\right\|^{2}\right) \\
& -\max _{\substack{\left[\mathbf{x}_{1}\right] \in \mathbf{x}^{d_{c}} \\
x_{k}=-1}} \star\left(\sum_{l \in \zeta_{n} \backslash k} \frac{x_{l}}{2} \ell_{c_{n} \leftarrow u_{l}}-\frac{1}{2 \sigma^{2}}\left\|y_{n}-\mathbf{h}^{[n] T} \mathbf{x}^{[n]}\right\|^{2}\right) .
\end{aligned}
$$




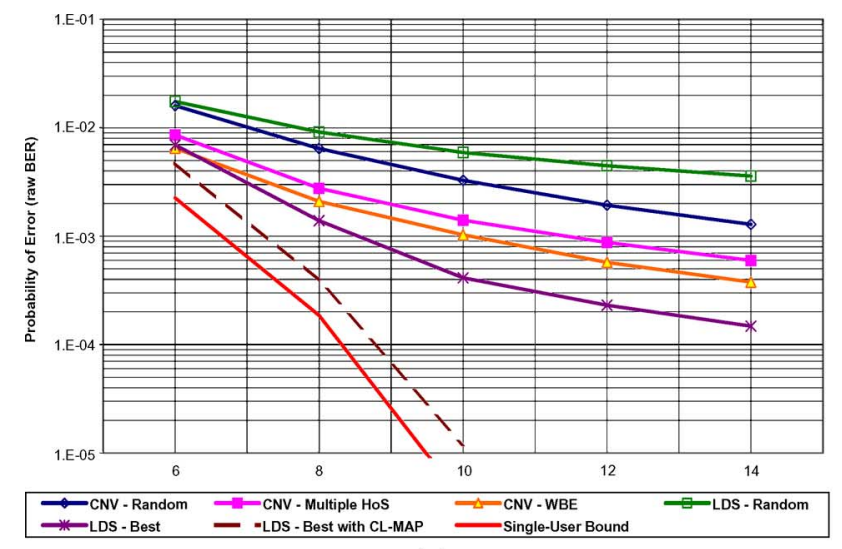

(a)

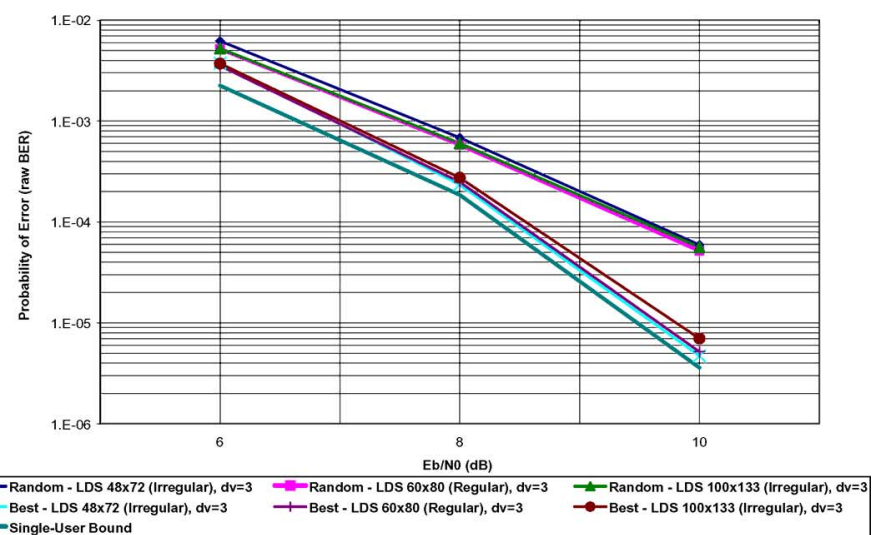

(b)

Fig. 3. Performance of LDS structures for DS-CDMA systems. (a) Comparison of different signatures structure with MMSE-PPIC. (b) large-system LDS schemes.

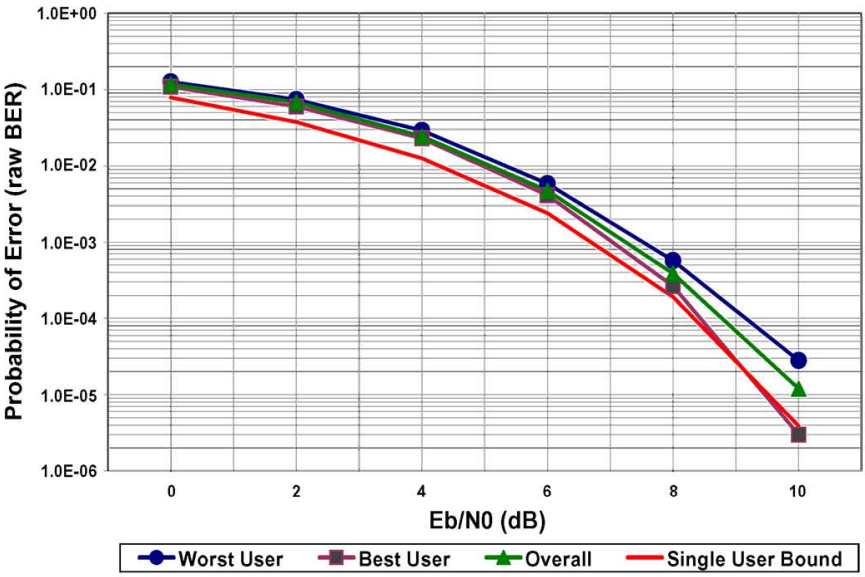

(a)

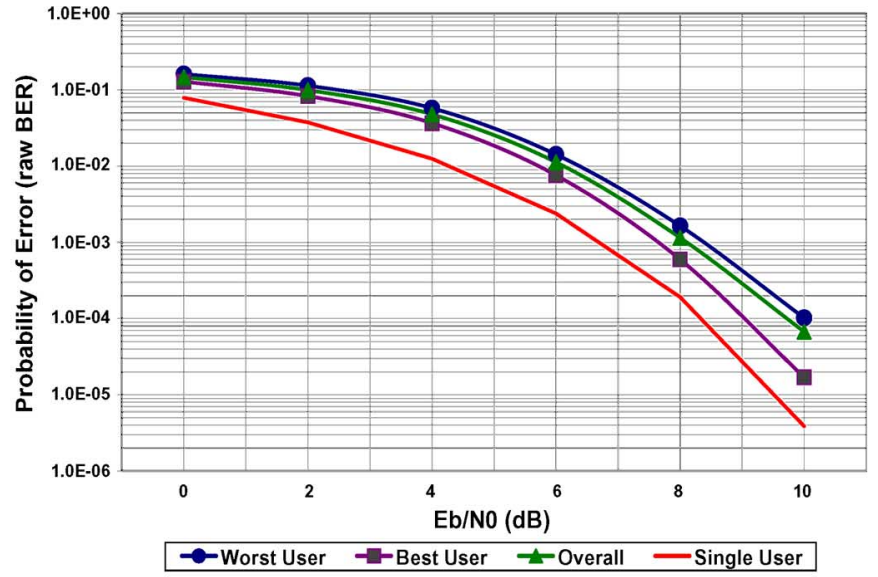

(b)

Fig. 4. Performance behavior of users in LDS structure. (a) LDS $12 \times 16$ (133\% loaded). (b) LDS $12 \times 24$ (200\% loaded).

penalty of slightly more complex signature the MMSE-based PPIC), it is shown superior to the conventional structure with any optimized signatures and its performance approached the single-user bound with penalty of $1.17 \mathrm{~dB}$ at BER of $10^{-4}$.

Furthermore, we also designed the LDS schemes for largesystem, where the number of users is more than 75 with different number of chips. From Fig. 3(b), the performance of different LDS schemes, using best-found signatures, with similar loading condition is shown to be similar. Notice, LDS for large-system perform even better, at higher SNR, than the small-system (e.g., LDS $12 \times 16$ ), especially when some degree of irregularity is imposed on the underlying factor-graph of the LDS schemes. Observing the performance of the LDS structure with the bestfound signatures, we conclude that, when we allow some irregularity and due to unit-energy-constraint of a user, the user's energy at some particular chip (where $d_{c}$ at this chip is lower than the average) dominate other interferers and make this user easier to be detected.

To gain more insight to the LDS structure, we also present another set of results that are showing the performance of individual users. Fig. 4(a) and (b) presents the performance of worst user and best user in LDS $12 \times 16$ and LDS $12 \times 24$, respectively. They show that some users will have weaker performance than other users. To be more precise, we can see that at low SNR region the performance degradation is not as high as in the high SNR region. This behavior can be explained by observing the signal constellation at each chip and the dominating effect of noise at low SNR region.

\section{B. Robustness Against Unequal Receive Power}

Here, unequal average receive power scenario was simulated. Moreover, it should be noted that the proposed LDS scheme does not give equal multiuser efficiency or in other words it does not result in the same performance for all the users as can be seen from Fig. 4.

Fig. 5 shows the performance of the LDS $12 \times 24$ system with unequal receive power. The performance of the best user and the worst user from the system with perfect-power control (PPC) were compared with the ones without power control, i.e., the system experiences a near-far effect (NFE) problem. As aforementioned, we know that each user, due to their signatures, will have different performance depending on the constellation of those chips, over which that a particular user spreads its data. Therefore, in this condition, to analyze the system performance, the observation should be made from different user's point of view, i.e., the weak user and the strong user. 


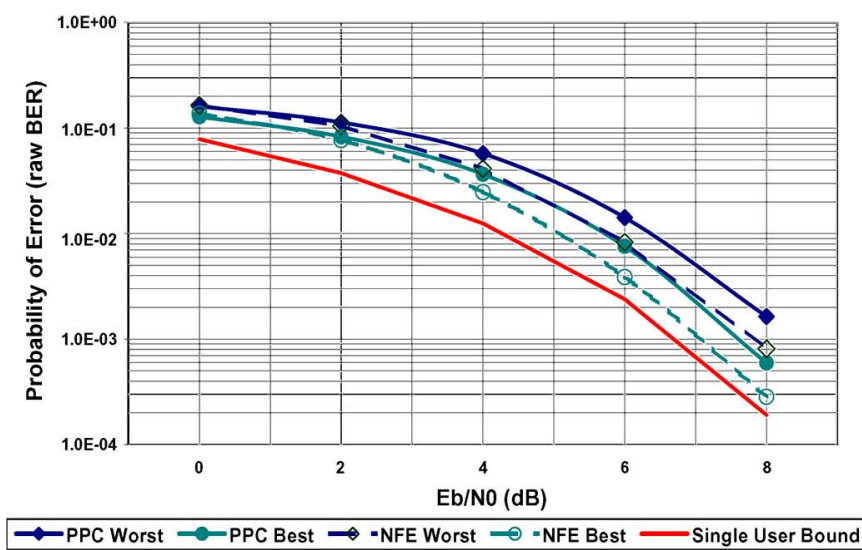

(a)

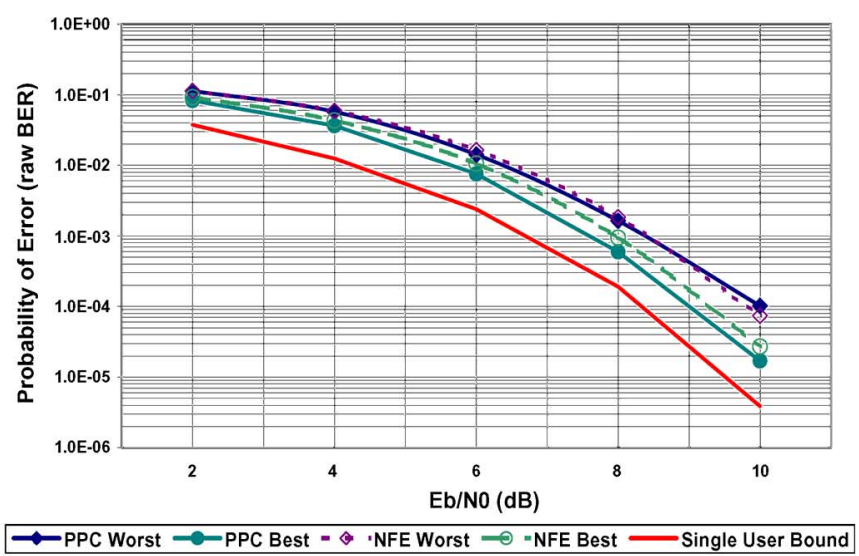

(b)

Fig. 5. Performance of LDS scheme under near-far effect condition. (a) From weak user point of view. (b) From stronger user point of view.

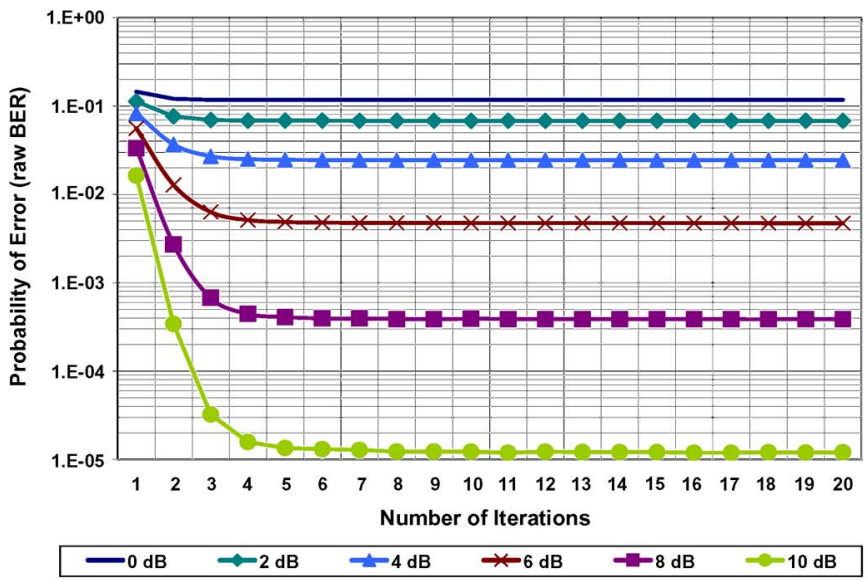

(a)

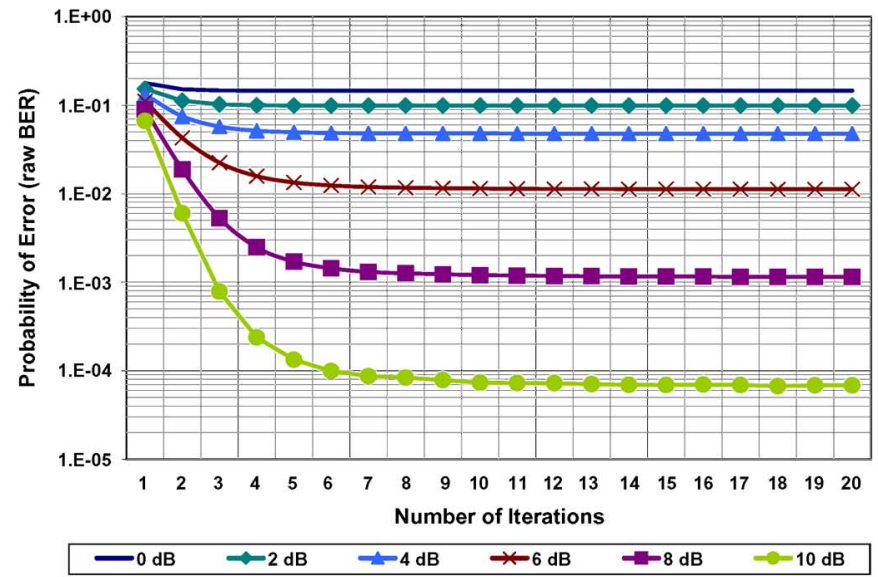

(b)

Fig. 6. Convergence behavior of different LDS schemes. (a) LDS $12 \times 16$. (b) LDS $12 \times 24$.

To simulate the unequal receive power scenario, we divide users into four groups of the same size, where the users from each group will be received at a power level that is $1 \mathrm{~dB}$ higher than previous groups. For instance, in LDS $12 \times 24$, there will be four groups of size 6 .

From weak users' point of view, the simulation suggests that the weak users will have their performance improved when there is strong user in the system compared and it is depicted in Fig. 5(a). But from the strong users' point of view, as Fig. 5(b) shows, they have a degraded performance. This phenomenon is due to the iterative joint processing being employed at our multiuser decoding algorithm. And exactly this phenomenon [27] that appears in the optimum detector for conventional CDMA systems, e.g., MAP detector.

\section{Convergence Rate}

In this subsection, the convergence rate of several LDS structures is investigated. By setting a maximum of 15 iterations, we can see the result in Fig. 6(a). In various LDS scheme being simulated, the performance will saturate after five iterations. The reason behind this is that, after certain threshold (iteration limit), the more iteration we run at the factor graph, the more correlated the messages become and, therefore, the extrinsic information become not very reliable to update the message. Without the independency of the extrinsic message, the performance will not increase any further or saturate. This problem has been addressed for LDPC decoding and the density evolution (DE) techniques have been used as the tool for its analysis [36] (see the references therein).

\section{CONCLUSION}

A novel LDS for synchronous CDMA have been proposed and investigated for communicaton over AWGN channel. The idea behind this proposal is basically to change the interference pattern being seen by each user; the proposed LDS structure limits the amount of interference occurred on each chip over which the user transmit a copy of his symbol. Having designed the LDS structure, the iterative chip-level SISO MUD algorithm, which is based on MPA, is proposed to efficiently approximate the APP of the symbols simultaneously. In this paper, MAP detection is done at chip-level, hence, its computational complexity with brute-force search is exponential to $d_{v}$, which is designed to be much smaller than $K$, instead of to $K$ 
as in conventional structure employing joint optimum MAP detection. Operating in memoryless Gaussian channel with BPSK modulation, the proposed structure and detection can approach very close to single-user performance without FEC coding in the overloaded condition of $200 \%$.

Note that, unlike conventional CDMA based employing multiuser detection, the LDS structure utilizing the proposed iterative chip-level MUD does not give the same multiuser efficiency for all users; some users will have better performance than others.

The extension of this new structure to multipath fading channel is still under investigation. It is understandable that the multipath fading channel will destroy the LDS structure; it creates multi short-cycles within the underlying graph and, hence, the proposed iterative MUD will not always converge to its true APP. Node-clustering can be utilized to minimize the number of short-cycles, yet it comes with the price of an added computational complexity. Therefore, a design of low-complexity receiver is imminent. Another way of tackling the frequency-selectivity of the channel is by employing LDS structure in multicarrier CDMA (MC-CDMA). The investigation and analysis of the proposed LDS structure of MC-CDMA will be presented in our other correspondences.

\section{REFERENCES}

[1] G. Golub and C. V. Loan, Matrix Computations, 3rd ed. Baltimore, MD: The John Hopkins Univ. Press, 1996.

[2] L. Welch, "Lower bounds on the maximum cross-correlation of signals," IEEE Trans. Inf. Theory, vol. 20, pp. 397-399, 1974.

[3] M. Rupf and J. Masey, "Optimum sequences multisets for synchronous code-division multiple-access channels," IEEE Trans. Inf. Theory, vol. 40, no. 4, pp. 1261-1266, Jul. 1994.

[4] P. Viswanath and V. Anantharam, "Optimal sequences and sum capacity of synchronous CDMA systems," IEEE Trans. Inf. Theory, vol. 45, no. 6, pp. 1984-1991, Sep. 1999.

[5] P. Cotae, "Transmitter adaptation algorithm for multicelullar synchronous DS-CDMA systems with multipath," IEEE J. Sel. Areas Commun., vol. 24, no. 1, pp. 94-103, Jan. 2006.

[6] J. Tropp, I. Dhillon, and R. Heath, "Finite-step algoriths for constructing optimal CDMA signature sequences," IEEE Trans. Inf. Theory, vol. 50, no. 11, pp. 2916-2921, Nov. 2004.

[7] R. Learned, A. Willisky, and D. Boroson, "Low-complexity joint detection for oversaturated multiple access communications," IEEE Trans. Signal Process., vol. 45, no. 1, pp. 113-122, Jan. 1997.

[8] F. Vanhaverbeke, M. Moeneclaey, and H. Sari, "DS/CDMA with two sets of orthogonal sequences and iterative detection," IEEE Commun. Lett., vol. 4, no. 9, pp. 289-291, Sep. 2000.

[9] F. Vanhaverbeke and M. Moeneclaey, "Optimal signature sets for oversaturated quasi-scalable direct-sequence spread-spectrum systems," IEEE Trans. Inf. Theory, vol. 51, no. 3, pp. 1136-1139, Mar. 2005.

[10] G. Rajappan and M. L. Honig, "Signature sequence adaptation for DS-CDMA with multipath," IEEE J. Sel. Areas Commun., vol. 20, no. 2, pp. 384-395, Feb. 2002.

[11] V. Khrishnamurthy, X. Wang, and G. Yin, "Spreading code optimization and adaptation in cdma via discrete stochastic approximation," IEEE Trans. Inf. Theory, vol. 50, no. 9, pp. 1927-1949, Sep. 2004.

[12] W. Santipach and M. Honig, "Signature optimization for CDMA with limited feedback," IEEE Trans. Inf. Theory, vol. 51, no. 10, pp. 3475-3492, Oct. 2005.

[13] H. Nguyen and E. Shwedyk, "A new construction of signature waveforms for synchronous CDMA systems," IEEE Trans. Broadcast., vol. 51, no. 4, pp. 520-529, Dec. 2005.

[14] S. Verdú, "Minimum probability of error asynchronous gaussian multiple-access channels," IEEE Trans. Inf. Theory, vol. 32, pp. 85-94, Jan. 1986.

[15] A. Duel-Hallen, M. Simon, and Z. Zvonar, "Multiuser detection for CDMA systems," IEEE Pers. Commun., vol. 2, pp. 46-58, Apr. 1995.
[16] M. Moshavi, "Multiuser detection for DS-CDMA communications," IEEE Commun. Mag., vol. 34, no. 10, pp. 124-136, Oct. 1996.

[17] S. Verdú, Multiuser Detection. New York: Cambridge Univ. Press, 1998.

[18] R. Lupas and S. Verdú, "Linear multiuser detectors for synchronous code-division multiple-access channels," IEEE Trans. Inf. Theory, vol. 35, pp. 123-136, Jan. 1989.

[19] M. Tsatsanis, "Inverse filtering criteria for CDMA systems," IEEE Trans. Signal Process., vol. 45, pp. 102-112, Jan. 1997.

[20] U. Madhow and M. Honig, "Mmse interference suppression for directsequence spread-spectrum CDMA," IEEE Trans. Commun., vol. 32, pp. 3178-3188, Dec. 1994

[21] H. Poor and S. Verdu, "Probability of error in MMSE multiuser detection," IEEE Trans. Inf. Theory, vol. 43, pp. 858-871, May 1997.

[22] M. Varanasi, "Group detection for synchronous gaussian code-division multiple-access channels," IEEE Trans. Inf. Theory, vol. 41, pp. 1083-1096, Jul. 1995.

[23] S. Buzzi, M. Lops, and G. Ricci, "A new group detection strategy for DS-CDMA systems," in Proc. Int. Symp. Inf. Theory, 2000, p. 357.

[24] A. Kapur and M. Varanasi, "Multiuser detection for overloaded CDMA systems," IEEE Trans. Inf. Theory, vol. 49, no. 7, pp. 1728-1742, Jul. 2003.

[25] A. Duel-Hallen, "Decorrelating decision feedback multiuser detector for synchronous code-division multiple-access channels," IEEE Trans. Commun., vol. 41, pp. 285-290, Feb. 1993.

[26] M. Varanasi, "Decision feedback multiuser detection: A systematic approach," IEEE Trans. Inf. Theory, vol. 45, no. 1, pp. 219-240, Jan. 1999.

[27] M. Varanasi and B. Aazhang, "Near-optimum detection for synchronous code-division multiple-access system," IEEE Trans. Commun., vol. 39, pp. 725-736, May 1991.

[28] A. Viterbi, "Very low rate convolutional codes for maximum theoretical performance of spread-spectrum multiple-access channels," IEEE J. Sel. Areas Commun., vol. 8, pp. 641-649, May 1990.

[29] D. Divsalar, M. Simon, and D. Raphaeli, "Improved parallel interference cancellation for CDMA," IEEE Trans. Commun., vol. 46, no. 2 , pp. 258-268, Feb. 1998.

[30] J. Luo, "Near-optimal multiuser detection is synchronous cdma using probabilistic data association," IEEE Commun. Lett., vol. 5, no. 9, pp. 361-363, Sep. 2001.

[31] L. Ping, "Interleave-division multiple access and chip-by-chip iterative multiuser detection," IEEE Commun. Mag., vol. 43, no. 6, pp. S19-S23, Jun. 2005.

[32] R. Gallager, "Low-density parity check codes," IRE Trans. Inf. Theory, vol. 8, pp. 21-28, Jan. 1962.

[33] F. Kschischang, B. Frey, and H. Loeliger, "Factor graphs and the sum-product algorithm," IEEE Trans. Inf. Theory, vol. 47, no. 2, pp. 498-519, Feb. 2001

[34] J. Boutros and G. Caire, "Iterative multiuser joint decoding: Unified framework and asymptotic analysis," IEEE Trans. Inf. Theory, vol. 48, no. 7, pp. 1772-1793, Jul. 2002.

[35] D. Mackay, Encyclopedia of Sparse Graph Codes [Online]. Available: http://www.inference.phy.cam.ac.uk/mackay/codes/data.html

[36] T. Richardson and R. Urbanke, "The capacity of low-density parity-check codes under message-passing decoding," IEEE Trans. Inf. Theory, vol. 47, no. 2, pp. 599-618, Feb. 2001.

[37] B. Hochwald and S. Brink, "Achieving near-capacity on multiple-antenna channel," IEEE Trans. Commun., vol. 51, no. 3, pp. 389-399, Mar. 2003.

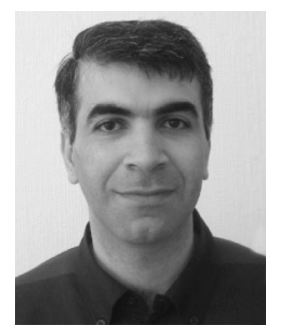

Reza Hoshyar (M'03) received the B.S. degree in communications engineering and the M.S. and Ph.D. degrees, both in mobile communications, from Tehran University, Tehran, Iran, in 1991, 1996, and 2001, respectively. He received the top and second rank awards for his B.S. and M.S. degrees from Tehran University.

From 2002 to June 2006, he has been a Research Fellow in the Mobile Communications Research Group, Center for Communication Systems Research (CCSR), University of Surrey, Surrey, U.K During this period, he has been actively involved in IST European projects MUMOR, STRIKE, and WINNER. Currently, he is a Senior Research Fellow at CCSR and participating as the technical work package leader in IST European project FIREWORKS, focusing on advanced digital signal processing, coding and modulation, and scheduling techniques for relaying, and cooperative communications in OFDM/OFDMA systems. 


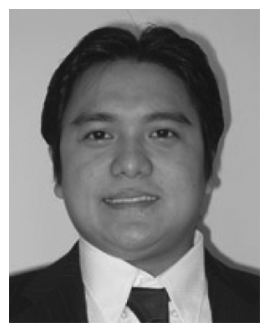

Ferry P. Wathan (S'01) received the B.S. (with distinction) and M.S. degrees, both in electrical engineering, from Bandung Institute of Technology, Indonesia, in 1999 and Dresden University of Technology, Germany, in 2002, respectively. He is currently pursuing the Ph.D. degree in mobile communications at the University of Surrey, Surrey, U.K.

His research interests include information theory, advanced multiuser detection and decoding, and stochastic processes.

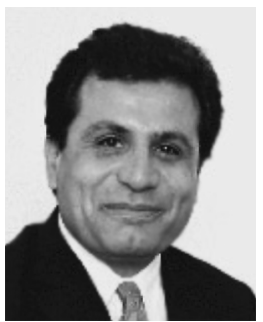

Rahim Tafazolli is a Professor of mobile/personal communication and Head of Mobile Communications Research Group, University of Surrey, Surrey, U.K. He has been active in research for 20 years and has authored and coauthored more than 300 papers in referred international journals and conferences. He has been a consultant to many mobile companies.

Prof. Tafazolli has lectured and chaired a number of IEE Summer schools and IEEE workshops and conferences. He is the Founder and Chairman of IEE International Conference on 3rd Generation Mobile Communications. He is the chairman of e. mobility technology platform expert group tasked with defining the strategic research agenda for EU FP7 programme of research on mobile/wireless communications. 